\title{
Partial Volume Effect
}

National Cancer Institute

\section{Source}

National Cancer Institute. Partial Volume Effect. NCI Thesaurus. Code C94947.

The smearing out of a scanned object's true activity concentration that should appear at each pixel (voxel) into a wider displayed pattern because of the lack of perfect scanner resolution for small objects (i.e. having a dimension near or less than the scanner's full width at half maximum of its point spread function). 